\title{
Co-operative Extended Kohonen Mapping (EKM) for Wireless Sensor Networks
}

\author{
Zenon Chaczko, Perez Moses, and Christopher Chiu \\ Faculty of Engineering \& IT, University of Technology, Sydney, Australia \\ zenon.chaczko@uts.edu.au, zindpm@gmail.com, christopher.chiu@uts.edu.au
}

\begin{abstract}
This paper discusses a methodology to manage wireless sensor networks (WSN) with self-organising feature maps, using co-operative Extended Kohonen Maps (EKMs). EKMs have been successfully demonstrated in other machine-learning contexts such as learning sensori-motor control and feedback tasks. Through a quantitative analysis of the algorithmic process, an indirect-mapping EKM can self-organise from a given input space, such as the WSN's external factors, to administer the WSN's routing and clustering functions with a control parameter space.

Preliminary results demonstrate indirect mapping with EKMs provide an economical control and feedback mechanism by operating in a continuous sensory control space when compared with direct mapping techniques. By training the control parameter, a faster convergence is made with processes such as the recursive least squares method. The management of a WSN's clustering and routing procedures are enhanced by the co-operation of multiple self-organising EKMs to adapt to actively changing conditions in the environment.
\end{abstract}

Keywords: Software Engineering, Extended Kohonen Wireless Sensor Networks (WSN), Sensor Actor Networks (SANET).

\section{Introduction}

The governance of wireless networks, particularly with WSNs, is important in managing the routing and clustering mechanisms in a dynamic and volatile environment [2]. Unlike static wired networks, the reliability and guarantees on communication stability can never be assured. Furthermore, the dependency on nodes to follow through on message relaying means that the failure of one node may lead to an entire branch losing total connectivity. Another main aspect is security, as the security within the network can be prone to denial-of-service attacks or technological espionage such as 'packet sniffing'. These particular domain concerns, among many more which are inherent with wireless networking, require the wireless network structure to adapt to changing environmental concerns to ensure the network's continual stability and robustness [10].

As with all open systems, wireless sensor network communications is inherently dynamic, such that the focus in achieving optimum conditions in a given environment is to determine the efficient routing path through the network. 
Modelling the domain-level conditions in a WSN is complex in nature, with a multitude of variables that influence the optimum routing condition. Transforming the multi-dimensional concerns to a single map is a formative approach to reduce the problem space into a single-dimensional map, in which the routing conditions can be established for the network.

The proposition for an alternate feature map approach is to be used for wireless sensor network management concerns, through co-operative EKMs with indirect mapping [8]. An indirect-mapping EKM approach is novel to existing direct-mapping methods by utilising the following techniques [11]:

\section{- Direct-Mapping Approaches}

Direct-mapping involves creating a Self-Organising Feature Map (SOM) that maps a sensory input directly to the node's stimuli in the wireless network. This would include parameters such as signal-to-noise ratios, battery charge levels and available bandwidth. Therefore, Direct-mapping methods map the continuous sensory stimuli space to discrete clustering or routing directives for each node, as seen from a different perspective.

\section{- Indirect-Mapping Approaches}

Indirect-mapping requires mapping the continuous sensory stimuli space to the node clustering or routing directive as an end result. The determination of the quality of clustering and routing approaches can be achieved through evaluating the cluster set data with validity matrices such as Dunn's Separation Index, Calinski-Harabasz and Davies-Bouldin's indexing methodologies [3]. Hence, the indirect-mapping approach maps sensory stimuli indirectly to a node clustering or routing directive with the utilisation of control parameters.

\section{Examining Co-operative EKMs}

The directives given to a node in a wireless sensor network's control space is formed as a discrete set of commands to be used by reinforcement learning algorithms [7,11], or at the minimum level, pre-defined static rules. In recent years, autonomous agent research in dynamic systems theory and reinforcement learning propose the operation of such directives in a continuous control space [7], to allow the indirect-mapping method to provide finer directive decisions than in direct mapping. Focussing on the flexibility and precision in sensory stimuli control is imperative in a wireless network domain where external environmental factors directly affect the network's robustness and reliability.

In traditional contexts, SOM or EKM in passive learning control is established and documented by Ritter [11]. However, the inconsequential problem of combining multiple SOMs or EKMs for sophisticated system control is a potential area of study. If the sensory control is insufficiently clarified, the routing or clustering decision made by a wireless node may be unexpected or undesirable, leading to a potential inertia to route data back to the sink or wireless gateway $[7,8]$. When SOMs or EKMs are established in the weighted-sum ensemble, a similar problem of inertia also takes place. 
To solve the problem of routing inertia, the combinational approach with cooperative EKMs will be applied to wireless sensor networks [8]. The co-operation and competition of multiple EKMs that similarly self-organise can enable a non-holonomic wireless node to optimise its routing and clustering choices in unexpected changes in its environment. In contrast, a node managed by the weighted-sum ensemble method will approach a routing inertia, even though the wireless network also implements a continuous sensory control space.

The environmental concern for a given wireless network domain can be summarised in the following statement tasks shown in Figure 1 on the following page; of which the environmental concerns deal with external factors such as sensory stimuli and node conditions [9]:

- For an initial state described by the input vector $U(0)$ in input space $U$;

- Adapt a new clustering or routing sequence of control vectors $c(t), t=$ $0, \ldots, T-1$ in the sensory control space $C$;

- With the resultant goal state elaborated by $u(T) \in U$ that adapts the network structure for a desired objective or target state.

The following algorithmic categories have been considered for an adaptive wireless sensor network environment which suits the above statement tasks:

\section{- Feature Mapping}

By using a SOM proposed by Kohonen [5], such as the Extended Kohonen Map (EKM) [12], the map self-organises to partition continuous sensory space into discrete regions. The feature map's generalisation capability arises from its self-organisation during training [6], such as when every node in the WSN is effectively trained to map a localised sensor region. This approach increases the sensory representation's resolution in the frequently encountered stimuli regions [6]. This conduct reflects biological sensory perceptions where frequent practice leads to better predictive capability of common, anticipated events.

\section{- Multivariate Regression}

An alternative approach formulates the statement task as a non-linear multivariate regression problem. Uninterrupted mapping from $U$ to $C$ is done by training a multilayer perceptron (MLP), which offers possible generalisation capability $[3,10,12]$. The main disadvantage prior to training the network is that training samples must be collected for each time step ' $t$ ' to define quantitative error signals. As this sampling process can be very tedious and computationally difficult, it is solved with the reinforcement learning approach by providing a qualitative success or failure feedback only at the end of the executing control sequence [4].

\section{Experimental Framework}

The experiment to evaluate the effectiveness of co-operative EKMs on WSNs is conducted using the MATLAB environment originally developed by 


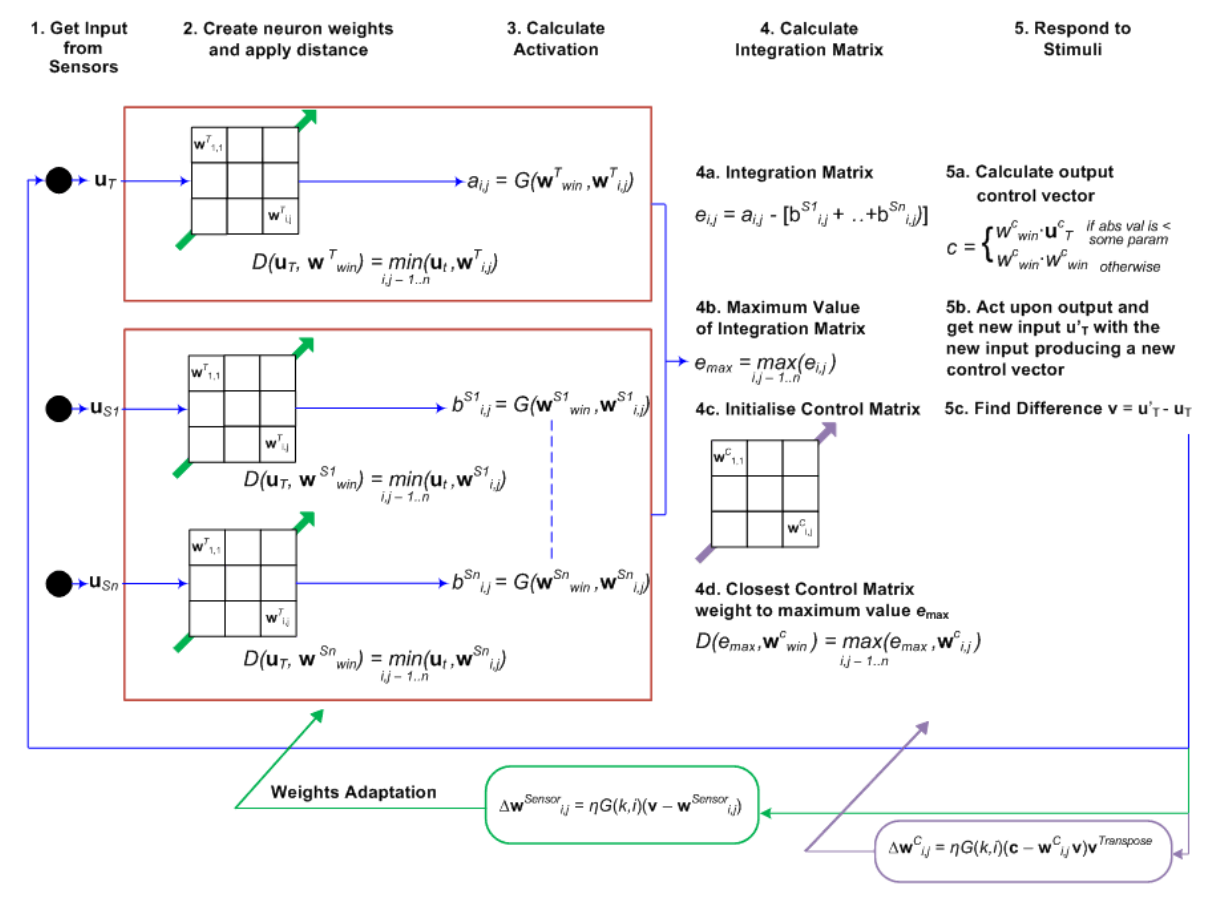

Fig. 1. Diagram of Co-operative EKM Functions

Chaczko, et al in 2003 [1]. The simulation framework, depicted in Figure 2 on the current page, allows for convenient monitoring and tracking of WSN events by programing event trajectories in the network field. The experiment is completed with the following methodology:

1. A population of $n$ nodes is distributed randomly using the Fast MersenneTwister method, in a two-dimensional network area of $100 \mathrm{~m} \times 100 \mathrm{~m}$. The total node population sampled include:
(a) 100 ;
(e) 2000;
(b) 250;
(f) 3000 ;
(c) 500;
(g) 4000;
(d) 1000;
(h) 5000 nodes.

2. An event trajectory is executed from a point in the network area; of which the test path can take the following courses:

(a) Linear Path

A linear path consists of an event trajectory where the entry and exit point from the network area consists of a constant gradient level.

(b) Arc-formation Path

An arc-formation path consists of an event trajectory where the entry 


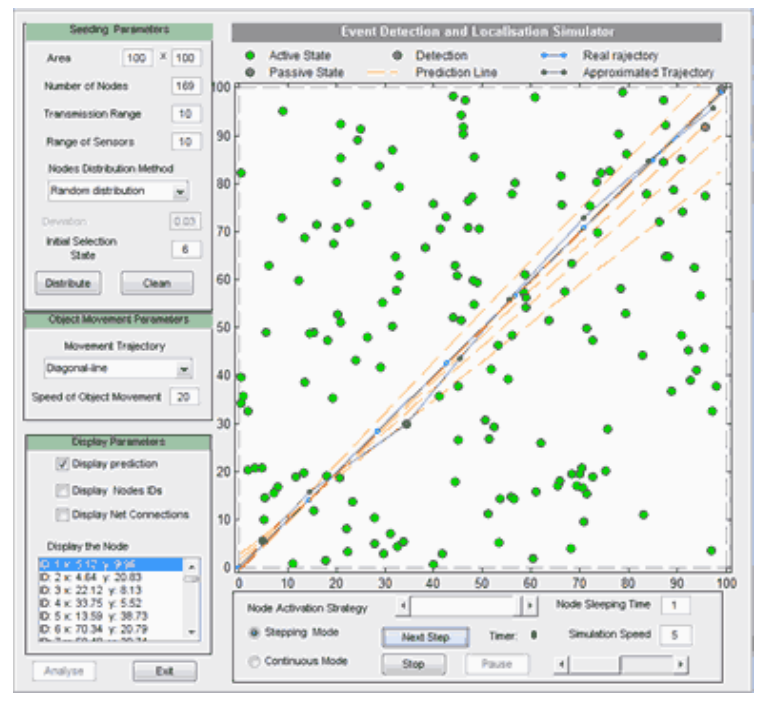

Fig. 2. Event Detection and Localisation Simulator Screenshot

and exit point of the network area will either be increasing or decreasing in the level of gradient, such that it forms a segment of a circle.

(c) Pseudo-random Path

A pseudo-random path using the Mersenne-Twister method combines elements of 2(a) and 2(b) at various points throughout the trajectory, until it reaches the exit point of the network area.

3. The algorithm selects the route from the node in range of the approximate trajectory to be established to the sink; such that the closer the algorithm is to calculating the event path, the more optimum the route will be to establish successful communications to the sink. The two algorithms that are assessed:

\section{(a) Linear Approximation}

Linear approximation estimates the approximate trajectory to determine the route path using general Euclidean geometry to calculate the final point of the event, based on the current nodes that are in contact with the event and tracing a path between the previous and current nodes.

(b) Co-operative EKMs

Co-operative EKMs use an indirect-mapping SOM map to train the control parameters in which to converge at the final trajectory point, in such a fashion to actively train the neural network to seek positive outcomes in reaching a final route from the trajectory's path to the sink.

4. The experiment is executed for 1000 iterations to calculate the mean rate of successful identification of the trajectory's target point. 
(a) A successful identification is where the final point is within a $98 \%$ confidence interval of the entire network area.

(b) Therefore, a maximum margin of error is determined to be an area of $2 \mathrm{~m} \times 2 \mathrm{~m}$ of where the final approximate point is calculated.

\section{Results}

The results shown in Figure 3 on this page demonstrate that in comparison to linear approximation and co-operative EKMs, the greatest promise in the final results can be achieved when pseudo-random trajectory tracking is required. While linear approximation of pseudo-random trajectories are expected to perform inadequately due to the inflexibility of the algorithm to accept large degrees in variation or change in the final result, co-operative EKMs demonstrate a noticeable improvement in the identification rate over linear approximation.

The analysis of co-operative EKMs in Table 1 on the next page, when assessed in terms of performance of pseudo-random tracking, requires more analysis into the algorithmic process. In particular, the thresholds established for determining positive or negative learning reinforcement is an issue that needs to be evaluated for an in-depth assessment. The tolerance levels used to calculate the thresholds is a delicate concern, as subtle variations in tolerance may yield undesirable results. As a case in point, reducing tolerance levels too far will result in the inflexibility of the algorithm to adapt to changes the event trajectory; the corollary is that generous tolerance levels will yield undesirable tracking results when noise or faulty nodes produce invalid sensory data.

As a consequence, the potential of Co-operative EKMs to identify events within a wireless sensor network show great promise; but as with all passive learning heuristic methods, a heuristic ensemble approach is required to train
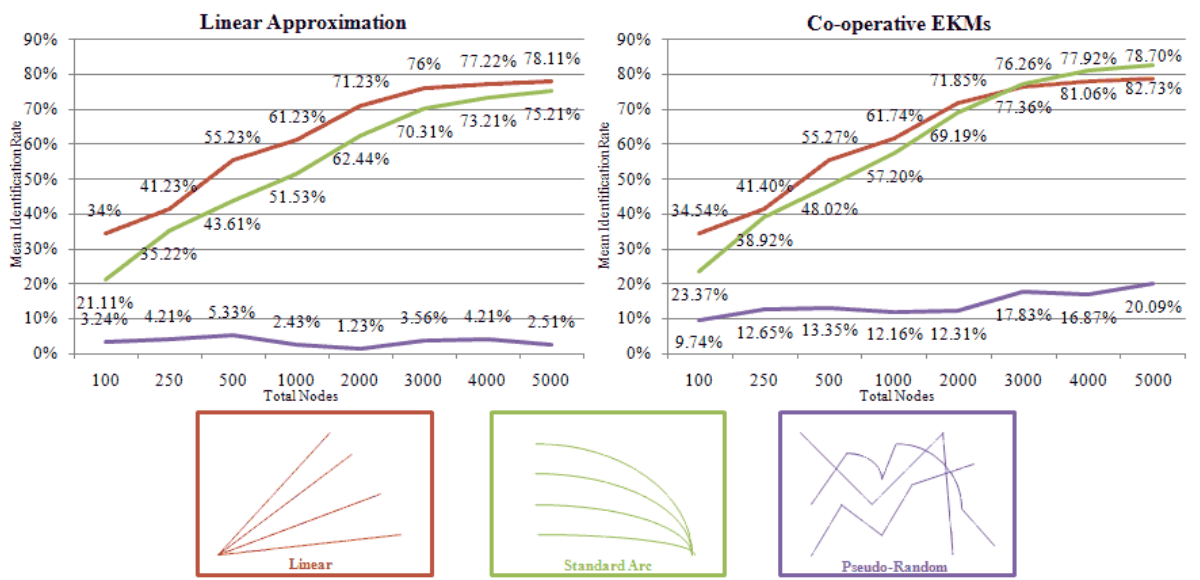

Fig. 3. Accuracy of Target Identification: Experimental Results 
Table 1. Comparative Assessment and Evaluation

\begin{tabular}{|c|c|c|}
\hline $\begin{array}{c}\text { Quantitative } \\
\text { Assessment }\end{array}$ & $\begin{array}{c}\text { Linear approximation is limited } \\
\text { with small node numbers as the } \\
\text { sparse distribution is a poor fit } \\
\text { to the estimated euclidean } \\
\text { geometry path }\end{array}$ & $\begin{array}{c}\text { Demonstrate an indirect- } \\
\text { mapping EKM can provide } \\
\text { detection to optimise for local } \\
\text { obstruction) and global (target } \\
\text { seeking) concerns }\end{array}$ \\
\hline $\begin{array}{c}\text { Qualitative } \\
\text { Assessment }\end{array}$ & $\begin{array}{c}\text { Linear approximation cannot } \\
\text { compensate for path obstruction } \\
\text { or unpredictable movement } \\
\text { without further algorithmic } \\
\text { improvement }\end{array}$ & $\begin{array}{c}\text { Preliminary results show a } \\
\text { smoother and finer tracking } \\
\text { mechanism to monitor events in } \\
\text { real-time, compensating for } \\
\text { random events [8,10] }\end{array}$ \\
\hline
\end{tabular}

the algorithm to evaluate and determine the tolerance thresholds that are most suitable for the current conditions. The implementation of co-operative EKMs with alternative heuristic algorithms such as genetic programming will be considered to evaluate improvement in the mean identification rate.

\section{Conclusion}

An innovative method of adaptive wireless sensor network governance responsibilities with co-operative EKMs has been established through evaluation; the preliminary results demonstrate indirect-mapping EKM generates more proficient wireless network governance decisions than other local learning methods like direct-mapping EKM. With recursive least squares, the control parameters of the indirect-mapping EKM can be trained to allow rapid convergence and improved optimisation when compared to the gradient descent.

The experimental results show a positive determination of the positive capability of co-operative EKMs in wireless sensor network routing. The notable variability in the identification rate is a result in the need to improve the quality of training mechanisms to reinforce positive selection processes, so the aggregation of the final routing selection is optimal for the given scenario. In addition, the current data-sets in future experimental assessments will be based on physical test-bed environments, in order to create a real-world scenario for quantitative evaluation of target identification and routing.

While linear and arc trajectories can be easily predicted using co-operative EKMs, further experimental study is required to improve the routing identification results of pseudo-random tracking using the technique of unsupervised learning. There are limitations to the degree of success that can be achieved with passive learning methods, before active supervision is necessary to train the system to seek patterns in target behaviour and act accordingly using weighting functions. 


\section{References}

1. Chaczko, Z., Klempous, R., Nikodem, J., Nikodem, M.: Methods of Sensors Localization in Wireless Sensor Networks. In: Proceedings of the 14th Annual IEEE International Conference and Workshops on the Engineering of Computer-Based Systems, March 2007, pp. 145-152 (2007)

2. Ghosh, J., Nag, A., Howlett, R.J., Jain, L.C.: An Overview of Radial Basis Function Networks: Radial Basis Function Networks: New Advances in Design, pp. 1-36. Physica-Verlag, New York (2001)

3. Halkidi, M., Batistakis, Y., Vazirgiannis, M.: Clustering Validation Techniques. Intelligent Information Systems Journal 17(2-3), 107-145 (2001)

4. Kaelbling, L.P., Littman, M.L., Moore, A.W.: Reinforcement Learning: A Survey. Journal of Artificial Intelligence Research, 237-285 (1996)

5. Kohonen, T.: Self-Organizing Maps, 3rd edn. Springer, New York (2000)

6. Martinetz, T.M., Ritter, H.J., Schulten, K.J.: Three-dimensional Neural Net for Learning Visuomotor Co-ordination. IEEE Transactions on. Neural Networks, 131$136(1990)$

7. Millán, J.D.R., Posenato, D., Dedieu, E.: Continuous-Action Q-Learning. Machine Learning, 249-265 (2002)

8. Low, K.H., Leow, W.K., Ang Jr., M.H.: Task Allocation via Self-organizing Swarm Coalitions in Distributed Mobile Sensor Network. In: 19th National Conference on Artificial Intelligence, pp. 28-33 (2004)

9. Low, K.H., Leow, W.K., Ang Jr., M.H.: An Ensemble of Cooperative Extended Kohonen Maps for Complex Motion Tasks. Neural Computation 17(6), 1411-1445 (2005)

10. Pomerleau, D.A.: Efficient Training of Artificial Neural Networks for Autonomous Navigation. Neural Computing, 88-97 (1991)

11. Ritter, H., Schulten, K., Denker, J.S.: Topology Conserving Mappings for Learning Motor Tasks: Neural Networks for Computing. In: American Institute of Physics: 151st Conference Publication Proceedings, Snowbird, Utah, pp. 376-380 (1986)

12. Sharkey, A.J.C., Sharkey, N.E.: Combining Diverse Neural Nets. Knowledge Engineering Review, 231-247 (1997) 